\title{
Coalescence of Macroscopic Magnetic Islands and Electron Acceleration from STEREO Observation
}

\author{
Hong-Qiang Song (宋红强), ${ }^{1}$ Yao Chen (陈耀), ${ }^{1}$ Gang Li (李刚), ${ }^{2,1, *}$ \\ Xiang-Liang Kong (孔祥良), ${ }^{1}$ and Shi-Wei Feng (冯士伟) ${ }^{1}$ \\ ${ }^{1}$ Shandong Provincial Key Laboratory of Optical Astronomy and Solar-Terrestrial Environment, School of Space Science and Physics, \\ Shandong University at Weihai, Weihai, Shandong 264209, China \\ ${ }^{2}$ Department of Physics and CSPAR, University of Alabama in Huntsville, Huntsville, Alabama 35899, USA
}

(Received 30 October 2011; revised manuscript received 28 March 2012; published 27 June 2012)

\begin{abstract}
Magnetic reconnection is a fundamental plasma process. Recent theoretical studies and numerical simulations have suggested that electrons can be efficiently accelerated in contracting magnetic islands and when magnetic islands coalesce. Using data from the STEREO spacecraft, we report in this article the first observation of macroscopic magnetic-island coalescence and a possible splitting, and the associated electron acceleration. On 24 May 2010, two magnetic islands were observed by both the STEREO-A and STEREO-B spacecraft to propagate out along the current sheet behind a coronal mass ejection (CME) and merge. Electron acceleration to above $10 \mathrm{keV}$ is inferred through the observation of a type-III-like radio burst. The acceleration process occurred at a macroscopic scale, likely during the merging of the two magnetic islands. Our observation of the magnetic-island coalescence is supported by a 2.5-D axisymmetric magnetohydrodynamic simulation of CME in which the merging of post-CME magnetic islands is clearly identified.
\end{abstract}

DOI: 10.1103/PhysRevX.2.021015

\section{INTRODUCTION}

Magnetic reconnection is a fundamental plasma process. It is believed to occur at solar flares [1], substorms in the Earth's magnetosphere [2], and other astrophysical sites such as magnetized neutron stars [3]. Recently, in situ evidence of magnetic reconnection near 1 A.U. (which is approximately the mean Earth-Sun distance) has been reported by Xiao et al. [4], who studied the 3D magnetic-reconnection process in the Earth's magnetotail using observations from the four-spacecraft Cluster array (Cluster), and Retinò et al. [5] who examined reconnection in the turbulent solar wind downstream of the Earth's bow shock.

A huge amount of magnetic energy is released during reconnection, and reconnection sites are thought to be very efficient particle accelerators. Indeed, many authors [6,7] have examined the energy contained in the accelerated electrons $>20 \mathrm{keV}$ in large flares and found that they could contain $10 \%$ to $50 \%$ of the total energy released in the flares. Recently, Drake et al. [8] proposed a mechanism of electron acceleration at contracting magnetic islands. In this mechanism, electrons are accelerated by bouncing back and forth between two ends of a contracting magnetic island, similar to the first-order Fermi acceleration mechanism proposed in the 1970s [9-13] that was used to explain

\footnotetext{
*Corresponding author. gang.li@uah.edu
}

Published by the American Physical Society under the terms of the Creative Commons Attribution 3.0 License. Further distribution of this work must maintain attribution to the author(s) and the published article's title, journal citation, and DOI.
Subject Areas: Astrophysics, Plasma Physics

high-energy cosmic rays. Drake et al. [8] suggested that efficient electron acceleration can be obtained if a current sheet is volume filled by multiple magnetic islands. Fu et al. [14] also studied the process of electron acceleration during collisionless magnetic reconnection using a particle-in-cell (PIC) simulation. They found that, in $\mathrm{X}$-type regions, electrons are more often accelerated through reflections and, in O-type regions, electrons are accelerated by a reconnection electric field as they are trapped in the magnetic island. These results are in general agreement with later simulations by Oka et al. [15], who noted that O-type regions can be more efficient in accelerating electrons at coalescing magnetic islands.

In situ observation of electron energization at magnetic islands has been reported by Chen et al. [16], who used the Cluster observations to show that energetic electrons are correlated with low-density regions within magnetic islands during reconnections in the Earth's magnetosphere. These authors noted that the presence of energetic electrons in the energy range of 35-94 keV coincides nicely with magnetic islands encountered by Cluster, providing the first in situ evidence of electron acceleration at reconnecting magnetic islands. Wang et al. [17] also reported in situ observations of a secondary magnetic island in an ion-diffusion region and associated energetic electrons using the Cluster array.

Unlike in situ observations in the Earth's magnetosphere, remote observations of magnetic-island-like structures at solar flare sites are rare. This is partly because, at a distance of 1 A.U., the angular resolution of a flare is too small. Nevertheless, recent simulations [18] based on a 2.5-D (two dimensions in configuration space and three dimensions in velocity space) PIC model have been 
put forth to investigate radio emissions generated during tearing and coalescence processes at the current sheet in a flare. The simulation of Karlický et al. [18] showed that drifting pulsating structures observed in solar radio spectra likely occur right before interacting plasmoids coalescence into larger ones, indicating efficient electron accelerations induced by the plasmoid interaction.

While solar-flare sites are perhaps too small to permit direct observation of magnetic islands, observations of these structures at a much larger scale, especially after the explosion of coronal mass ejections (CMEs), do exist. Indeed, Ko et al. [19] and Lin et al. [20] both reported the identification of current sheets and magnetic islands (called "blobs") using C2 and C3 images from the Large Angle and Spectrometric Coronagraph (LASCO) aboard the Solar and Heliospheric Observatory (SOHO). More recently, Riley et al. [21] studied bursty reconnection following a CME eruption using a numerical magnetohydrodynamic (MHD) simulation. The formation and evolution of blobs along the post-CME current sheet from their numerical simulations compared well with LASCO SOHO observations.

Besides being found in the current sheet behind a CME, blobs have also been found to exist in current sheets of coronal streamers. Recent 2.5-D axisymmetric numerical simulation by Chen et al. [22] has shown that blobs can emerge naturally from the current sheet near the cusp region of a streamer as a result of the dynamical equilibrium between the plasma expansion and the magnetic-field confinement. Following [19,20,23], we have recently examined various properties of CME- and streamer-related blobs [24,25]. In some events, we identified multiple blobs in a time span of several to a few tens of hours. In these observations, blobs, understood as the products of magnetic reconnections along the associated current-sheet structures, have a size similar to the width of the currentsheet ray structure, and therefore are macroscopic in scale. The work of Sheeley et al. [26] and McKenzie and Savage [27] also reports observations of magnetic islands, at postflare loops, identified as "tadpoles." (However, see Savage et al. [28] for an alternative interpretation.)

Using a 2-D particle-in-cell simulation, Oka et al. [15] showed that the coalescence of magnetic islands can provide a natural site for an efficient electron acceleration. In their simulation, most of the acceleration occurs at or near the merging points; therefore, the acceleration efficiency is decided by the amount of the flux in the merging field. In this picture, magnetic islands need not be volume-filling as was proposed by Drake et al. [8]. Oka et al. [15] further suggest that such coalescence can occur at solar flares and lead to the observed hard X-ray at both the top and the foot points. Their simulation [15] contains no intrinsic scale and can be applied to converging macroscopic blobs as well.

In this paper, we present, to our knowledge, the first ever observational evidence of a macroscopic magnetic-island coalescence (and a possible magnetic-island splitting) along a current sheet behind a CME eruption and the associated electron acceleration.

\section{EVENT IDENTIFICATION AND ANALYSIS}

To identify blob events, we use the COR1 and COR2 coronagraph instruments [29] onboard STEREO-A and STEREO-B [30]. A blob is bright, high-density material that propagates outward along a raylike structure. The rays themselves are dense coronal plasma-sheet structures and are believed to be either current sheets behind coronal mass ejections or plasma sheets above streamers [25]. (See also $[19,20,24,31]$.) Blob events are often triggered by CMEs. Many are found to occur along the current sheet behind the CME. Some of them also occur along a nearby streamer plasma sheet which is disturbed by the ejection. In a few cases, blobs can be generated without a clear CME signature. For example, a blob can emerge after the tip of a streamer is disconnected from the rest of the streamer, a result of the streamer's slow but persistent expansion.

In identifying all multiple-blob events, we require that two or more blob structures are recognizable from the COR2 white-light images of STEREO-A. We have examined the period from 1 January 2007 to 31 August 2011 and found 45 multiple-blob events. The properties of these blobs are listed online [32]. According to their physical origins, we classify these 45 events into three types: type I denotes events in which blobs are observed along a CMEtrailing ray structure; type II denotes events in which blobs are observed along a preexisting streamer plasma sheet that is strongly disturbed by a nearby CME eruption; and type III denotes events in which blobs are caused by a streamer disconnection without CME involved. Among these 45 events, five showed signs of coalescence (see Table I). To confirm the coalescence, we also examined STEREO-B observations. Two of the five events did not have simultaneous STEREO-B observations.

Upon further examination of the remaining three events, event 3 has the most definite signs of blob coalescence from both STEREO-A and STEREO-B observations. In the following discussion, we focus on this event.

Event 3 occurred on 24 May 2010. The relative configurations of the STEREO-A, STEREO-B, and SOHO spacecraft are shown in Fig. 1. Also shown in Fig. 1 are the magnetic-field configurations obtained from the potentialfield-source-surface (PFSS) model [36] as seen from each of the three spacecraft. The red arrows in the figure point to the location of the diffuse bipolar structure from which the CME erupted. At 18:06 (on 23 May), LASCO/C2 observed a halo CME with an apparent linear speed of $258 \mathrm{~km} \mathrm{~s}^{-1}$. The CME was also observed in STEREO-A (STEREO-B) propagating outward with a linear speed of 362 (378) $\mathrm{km} \mathrm{s}^{-1}$. After the ejection, in the current sheet behind the CME, two blobs were generated (likely through tearing instability) in sequence. So event 3 was a type-I 
TABLE I. Candidates of blob-coalescence events as observed by the STEREO spacecraft from 1 January 2007 to 31 August 2011.

\begin{tabular}{|c|c|c|c|c|}
\hline $\begin{array}{l}\text { Event number } \\
\text { (and type) }^{\mathrm{a}}\end{array}$ & CME date, time ${ }^{b}$ & $\begin{array}{c}\text { CME } \\
\text { principal } \\
\text { angle }^{c}\end{array}$ & $\begin{array}{l}\text { Ray position angle, } \\
\text { date, and time }{ }^{\mathrm{d}}\end{array}$ & $\begin{array}{c}\text { Coalescence } \\
\left.\text { date, time, height (in } R_{\odot}\right)^{\mathrm{e}}\end{array}$ \\
\hline $1(\mathrm{III})^{\mathrm{f}}$ & 25 June $2008,18: 37$ & & 244, 25 June 2008, 18:37 & 26 June 2008, 05:07, 9.3 \\
\hline $2(\mathrm{I})^{\mathrm{fg}}$ & 13 September 2008, 02:37 & 101 & 94, 13 September 2008, 19:07 & 13 September 2008, 18:07, 10.0 \\
\hline $3(\mathrm{I})$ & 23 May $2010,17: 54$ & 79 & 58, 24 May 2010, 06:24 & 24 May $2010,08: 24,5.0$ \\
\hline 4 (II) & 28 October $2010,11: 24$ & 250 & 234,29 October $2010,15: 24$ & 30 October $2010,20: 39,11.0$ \\
\hline $5(\mathrm{I})$ & 26 November 2010, 08:24 & 242 & 231, 26 November 2010, 17:24 & 27 November 2010, 12:24, 9.0 \\
\hline
\end{tabular}

${ }^{a}$ Event number: Specifies the five events that showed signs of coalescence. Event types: type I: The blobs in the event are observed along a CME-trailing ray structure; type II: The event takes place along a preexisting streamer plasma sheet (the ray structure), which is strongly disturbed by a nearby CME eruption; type III: The event is caused by a streamer disconnection, and no CME is involved. ${ }^{\mathrm{b}}$ The date and time (in 24-hour convention) of the first COR2A appearance of each CME.

${ }^{\mathrm{c}} \mathrm{CME}$ principal angle (in degrees), as determined by the CACTUS project [33-35].

${ }^{\mathrm{d}}$ Ray position angle (in degrees) at $3 R_{\odot}$ and the corresponding measurement date and time.

eApproximate coalescence date and time, and the heliocentric height, from the COR2A observations.

${ }^{\mathrm{f}}$ These first two events are seen only by STEREO-A. The rest are seen by both STEREO-A and STEREO-B.

${ }^{\mathrm{g}}$ In this event, the blob is observed to merge into the leading CME ejecta.

event. Note that, because there was a nearby streamer to the north of the CME that interacted with the CME as the CME propagated out, there were some thoughts that event 3 could be a type-II event. However, upon careful examination, we have established that it is a type-I event.

Figures 2(a) and 2(b) show the morphology and the temporal evolution of the two coalescing blobs as observed by STEREO-A's COR1 and COR2 instruments and STEREO-B's COR1 and COR2 instruments. Figure 2(a) shows the white-light images, and Fig. 2(b) shows the running-difference images. The first blob appeared at

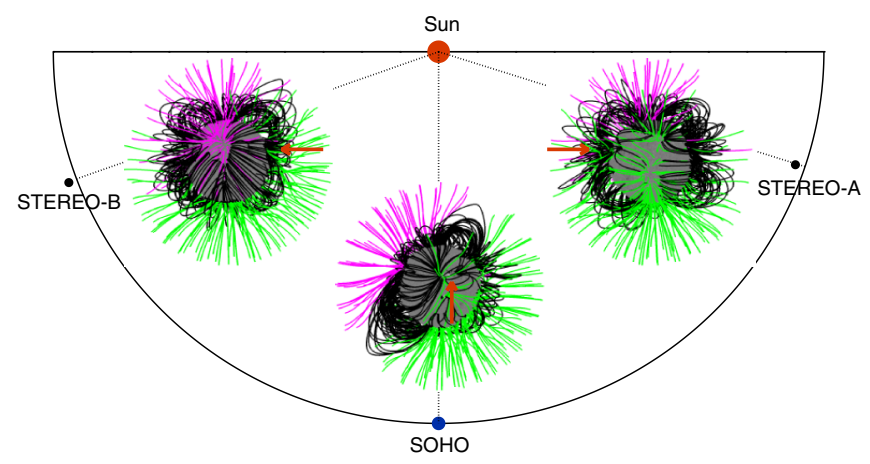

FIG. 1. Blob event 3 as observed from the three spacecraft STEREO-B, SOHO and STEREO-A, respectively. The CME preceding the merging of the two blobs occurred on 23 May 2010, and the blobs were observed on 24 May 2010. The longitudinal separation between the STEREO-A (STEREO-B) spacecraft and the SOHO spacecraft is $71.62^{\circ}\left(69.97^{\circ}\right)$. A halo CME that erupted from a diffuse bipolar structure at 18:06 on 23 May was observed by the LASCO instrument on SOHO. The CME was also observed in both STEREO-A and STEREO-B spacecraft. The magnetic-field configurations obtained by the PFSS model [36] as seen from STEREO-A, SOHO, and STEREO-B are also shown in the figure. The red arrows indicate the location of the diffuse bipolar structure from which the CME erupted. To the north of the CME is a nearby streamer (shown as closed field lines in black).
04:54 on 24 May 2010. It slowly moved upward and became elongated, as shown in the following several frames. Around 07:09, the elongation seemed to lead to a magnetic reconnection, and the center of the first blob split into two smaller ones. By 07:24, the first blob evolved into a "two-core" peanut shape (corresponding to the left drawing in Fig. 2(g). This can be clearly seen from the frame at 07:39 in Fig. 2(a) as well. Because of a time resolution of 15 minutes between the frames, a clear determination of when the splitting started and ended is difficult. The splitting may have been completed before 07:24 since the general morphology of the blobs does not change much between 07:24 and 07:39. Consequently, the splitting lasted only for up to 15 minutes. At 07:24, the length and width of the upper "daughter" blob were $0.41 R_{\odot}$ and $0.19 R_{\odot}$, respectively; the length and width of the lower daughter blob were $0.22 R_{\odot}$ and $0.17 R_{\odot}$, respectively. Compare these measurements with the "mother" blob's length of $0.72 R_{\odot}$ and width of $0.18 R_{\odot}$ at time 07:09. Considering the uncertainties of these measurements, either slight or no contraction occurred during the splitting.

From Figs. 2(a) and 2(b), we can also see that the second blob starts to appear in the view at 06:39. It moves faster (with a linear speed of $361 \mathrm{~km} \mathrm{~s}^{-1}$ ) than the first blob (with a linear speed of $187 \mathrm{~km} \mathrm{~s}^{-1}$ ); it is identified by the white arrows in the three frames of 07:39, 07:54, and 08:09. By 08:09, the second blob catches up to the bottom of the first blob. Afterward, the coalescence begins, as can be seen clearly in Fig. 2(a)'s 08:24 frame [corresponding to the middle drawing in Fig. 2(g)]. The coalescence continues for the next few frames (09:24, 09:39, and 09:54), and the combined magnetic island moves further up (with a linear speed of $326 \mathrm{~km} \mathrm{~s}^{-1}$ ), and expands sideways as well. The morphology of this joint magnetic island does not vary much until perhaps 10:09 and/or 10:24, when the upper outer part of the magnetic island appears to open up. (This opening up is further illustrated in Fig. 2(f) using 
(a)

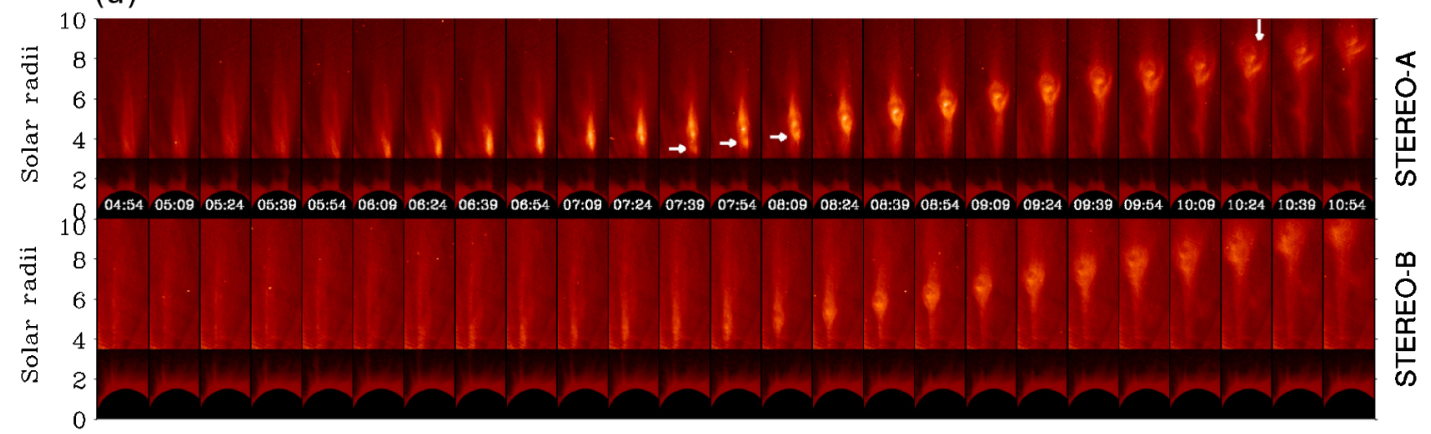

(b)

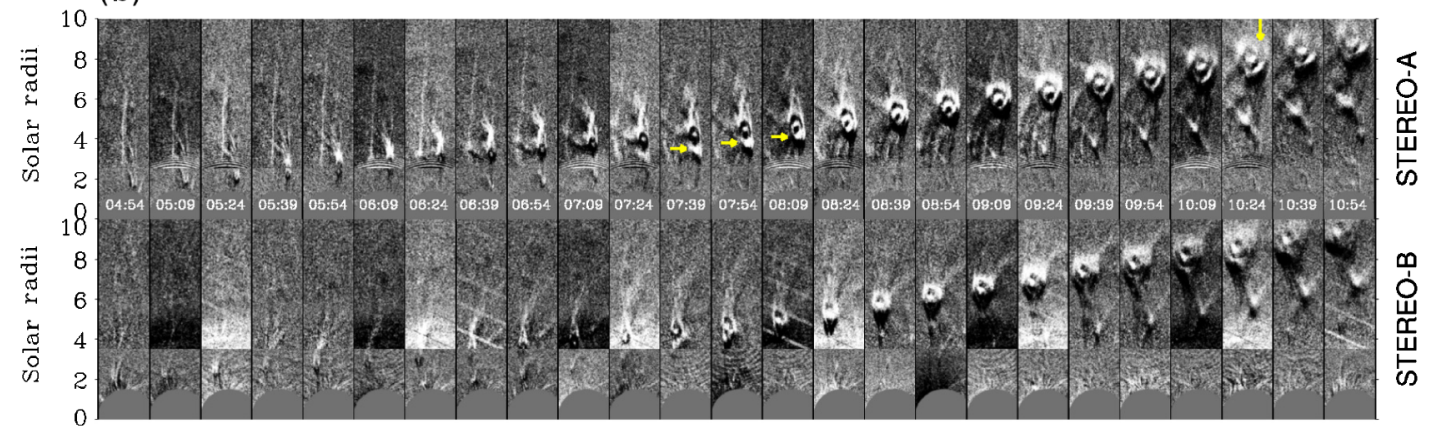

(c)

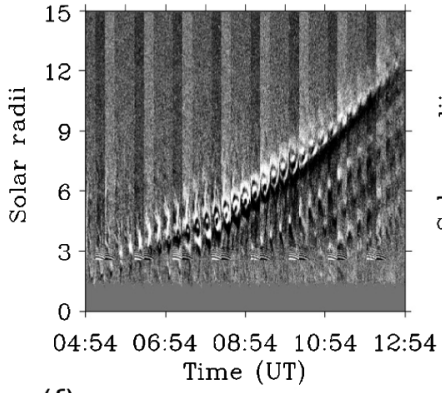

(f)

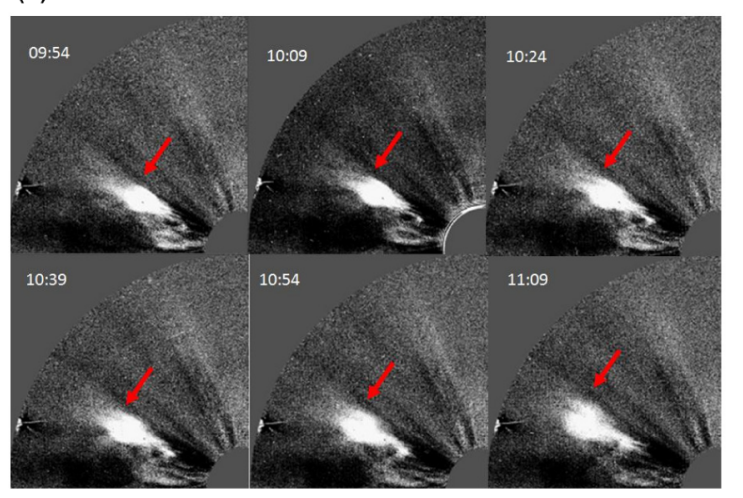

(d)

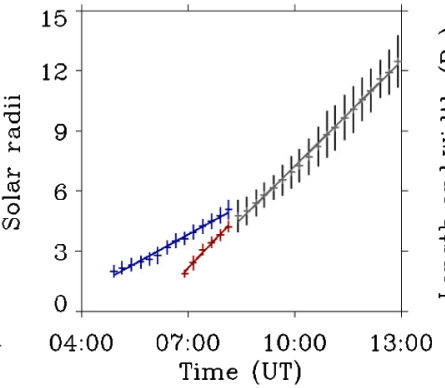

(g) (e)
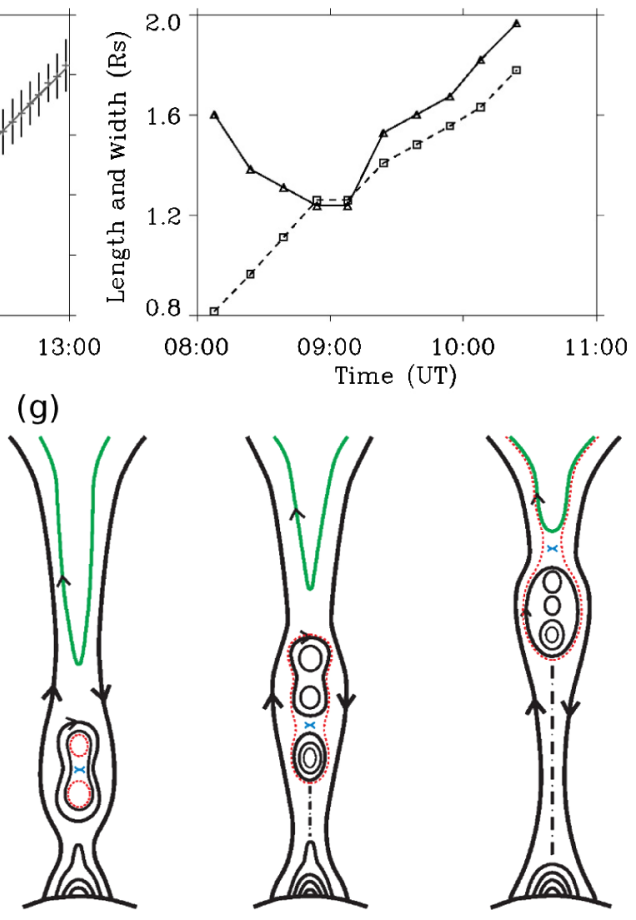

FIG. 2. (a),(b) The white-light and running-difference images of the magnetic blobs from STEREO-A and STEREO-B observations. The first blob evolved into a "two-core" peanut shape in the 07:39 frame. White arrows indicate the second blob moving and catching up to the first blob. Between 10:09 and 10:24, the upper right part of the blob appeared to open up (as indicated by the arrows), suggesting possible reconnection with the open magnetic-field lines lying above. (c) The running-difference image showing the merging process (left). (d) The height of the center of the blobs before and after they merge. (e) The length (solid line) and the width (dashed line) of the blobs during the merging phase. (f) The base-difference images showing the reconnection with the overlying magnetic field. The base image for these plots is from the frame with time 04:24. The opening-up phase is clearly seen from the 10:24 frame (and thereafter), although it is possible that it may have started in the 10:09 frame. (g) Drawings show the three reconnection processes in this event. Left: The breaking of the first blob into a two-core-peanut blob. Center: The merging of the two blobs. Right: The reconnection between the merged blob with the overlying magnetic field. Blue crosses mark the reconnection sites. Field lines before the reconnection are shown as black and green solid lines; field lines after the reconnection are shown as red dotted lines. 
the base-difference images.) We suggest that such an opening up is the result of magnetic reconnection between the blob and the overlying magnetic-field lines that trailed the CME [see the drawings of Fig. 2(g)], as indicated by the red arrows in Fig. 2(f). This opening up occurs shortly before the type-III-like radio burst (shown in Fig. 4), which was observed by the WAVES instruments on STEREO-A [37]. Note the spatial scale of the blobs: At time 10:24, the size of the blob is as large as $2 R_{\odot}$.

To better examine the merging process, we show, in Fig. 2(c), the running-difference images of the two blobs before and after the merging. We also plot in Fig. 2(d) the height of the centers of the blobs before and after the merging. The length of the vertical lines represents the size of the blobs. The lines for the first blob are blue; for the second blob, red; and for the merged blob, black. It can clearly be seen that the second blob moves faster than the first. After the merging, the merged blob moves more slowly than the second but faster than the first, as expected. Figure 2(e) plots the length (solid line) and width (dashed line) of the merged blob between 08:09 and 10:24. (See also Table II.) The merged blob is initially very elongated. As the merging continues, due to the difference in the speeds of the two blobs, the length of the merged blob decreases and its width increases. However, it is clear from Fig. 2(e) that the expansion dominates the contraction during the merging phase. Indeed, within the measurement uncertainties, no clear signatures of contraction can be identified during the merging phase.

As discussed by Drake et al. [8,38], contraction of magnetic islands can lead to very efficient electron acceleration. In our event, there was no contraction during the merging phase, and therefore the associated electronacceleration mechanism during the merging phase cannot be the one proposed by Drake et al. $[8,38]$. However, the merging process itself can efficiently accelerate electrons according to Oka et al. [15]. Indeed, Oka et al. suggested that, during the coalescence of magnetic islands, contraction plays a less important role than merging in energizing electrons [15]. We therefore still expect that electron acceleration occurred during the merging. We note here that the mechanism discussed in Drake et al. can be applied during the splitting phase (see discussions below), although, in our case, only one reflection point existed, instead of two as in the Drake et al. work [8]. We also point out that, since the blobs propagated out radially and were subject to expansion, any kind of acceleration had to be impeded because the expansion can decelerate particles. This deceleration is of adiabatic cooling, however, and is not as "efficient" as the acceleration caused by either the splitting or the merging process.

Figure 2(f) shows the base-difference images for six time frames between 09:54 and 11:09. The base image corresponds to the time frame of 04:24. While runningdifference images are best suited to track and examine the motions of the blobs, a base-difference image allows us to concentrate on transient features that emerge between frames. From Fig. 2(f), an opening up is clearly seen to emerge in the 10:24 frame. The opening up becomes more conspicuous in the next few frames, and, by 11:09, the blob has deformed considerably where some material seems "ready to be detached" from the upper side. We suggest that this opening up corresponds to the magnetic reconnection between the blob and the overlying magnetic field. Such a reconnection is driven by the upward motion of the blob and is a slow process (see discussions below). The exact starting time of this reconnection is hard to pin down. It is likely between 10:09 and 10:24. There is a slight sign of the opening up in the 10:09 frame, but it is not clear.

Finally, the drawings in Fig. 2(g) show the three reconnection processes in this event: the splitting of the first blob into a two-core-peanut blob, the merging of the two blobs, and the reconnection between the merged blob with the overlying magnetic field. Blue crosses indicate the reconnection sites. Field lines before the reconnection are shown as black and green solid lines, and the red dashed lines show field lines after the reconnection.

To better understand our observation, we also study the merging process of the blobs using a 2.5-D axisymmetric MHD simulation. We use a catastrophic flux-rope model [39-41] to simulate the CME eruption process. As shown in Fig. 3, the system is initially in a configuration consisting of a triple-streamer solar-wind system. Such a configuration is a result of heating the plasma that was contained by a prescribed quadrupole magnetic field. The heating process is simplified as a polytropic process with the polytropic index $\gamma$ set to 1.05. A flux rope characterized by a poloidal flux per radian of $\Phi_{p}=0.3 \Phi_{0}$ and a total toroidal flux of $\Phi_{\varphi}=0.334 \Phi_{0}$ emerges from the central bottom of the left streamer from $t=0$ to $t=2 \mathrm{~h}$.. The

TABLE II. The morphological change of the merged blob in event 3 from 08:09 to 09:54 on 24 May 2010.

\begin{tabular}{lcccccccc}
\hline \hline Time & $08: 09$ & $08: 24$ & $08: 39$ & $08: 54$ & $09: 09$ & $09: 24$ & $09: 39$ & $09: 54$ \\
\hline Height $^{\mathrm{a}}\left(R_{\odot}\right)$ & 4.73 & 5.02 & 5.31 & 5.68 & 6.11 & 6.40 & 6.83 & 7.20 \\
${\text { Length }\left(R_{\odot}\right)}^{1.60}$ & 1.38 & 1.31 & 1.24 & 1.24 & 1.53 & 1.60 & 1.67 \\
Width $\left(R_{\odot}\right)$ & 0.81 & 0.96 & 1.11 & 1.26 & 1.26 & 1.41 & 1.48 & 1.56 \\
\hline \hline
\end{tabular}

${ }^{\mathrm{a}}$ The height is measured from the center of the blob. 


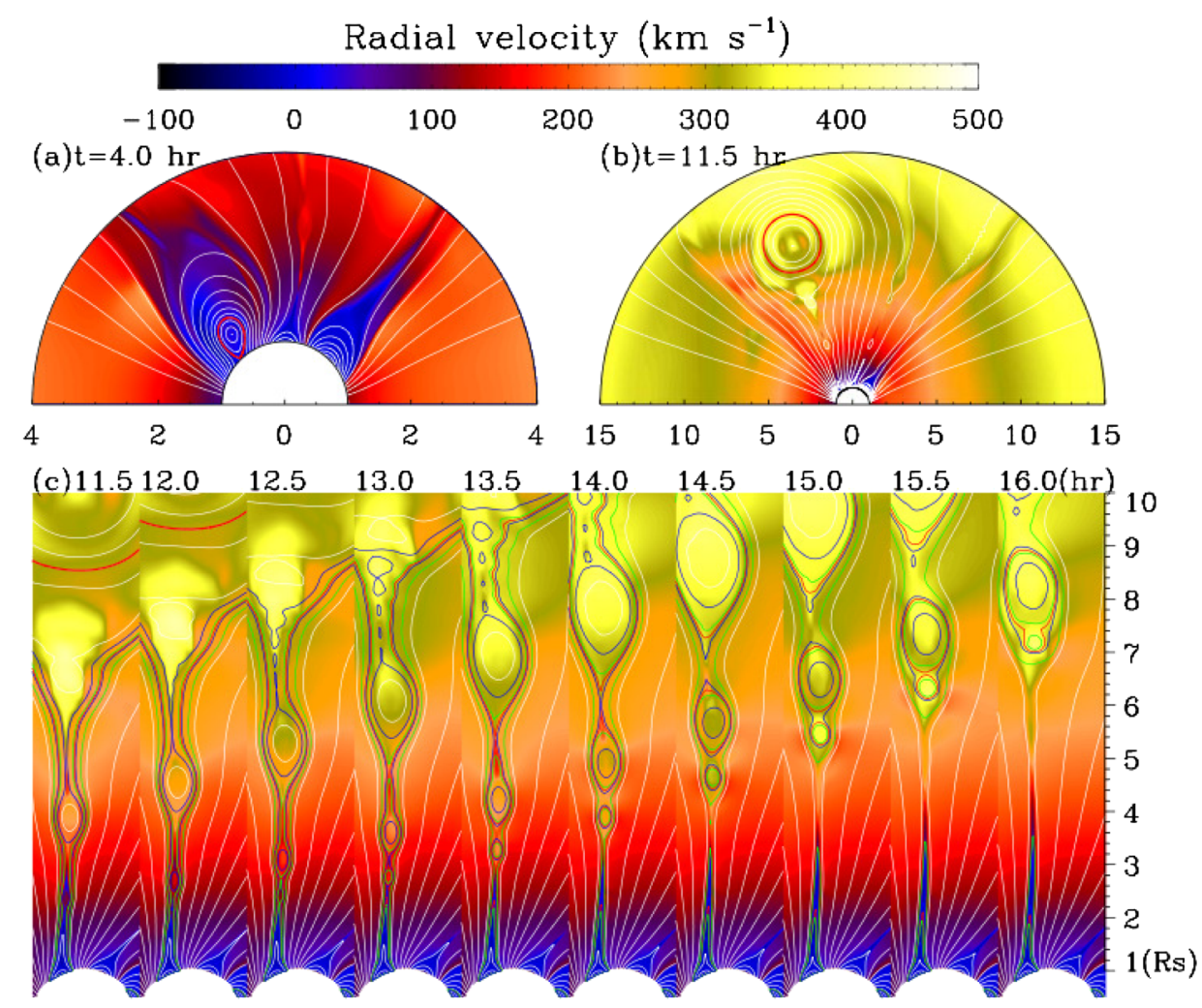

FIG. 3. A 2.5-D axisymmetric MHD simulation based on the catastrophic flux-rope model showing the generation and the merging of multiple blobs in the current sheet behind a CME eruption. (a),(b) Snapshots at times $t=4.0 \mathrm{~h}$. and $t=11.5 \mathrm{~h}$., respectively. White lines are magnetic fields. The colors depict the radial velocity, matching the legend across the top of the figure. The CME is modeled by a flux rope, represented by the red circles in (a),(b), that emerges from the bottom center of the leftmost streamer. (c) is a time sequence of 10 frames, 30 minutes apart, from $t=11.5$ to $t=16 \mathrm{~h}$. It represents a plot enlargement along the current sheet behind the CME. The width of the slide (i.e., the horizontal size) in each frame in (c) is $2 R_{\odot}$. Two animations of blob generation and coalescence corresponding to (a),(b) can be found online [32]. Note that, as can be seen from the online animations, blob formation and coalescence can also occur along the streamer plasma sheet that is strongly disturbed by the CME.

parameter $\Phi_{0}=4.023 \times 10^{13} \mathrm{Mx}$ is the unit of magnetic flux. The system further evolves. At time $t=4 \mathrm{~h}$., two hours after the flux rope emerges, the radial-velocity field from 1 to $4 R_{\odot}$ is shown as the contour map in Fig. 3(a) with the value of the velocity given by the color bar at the top of Fig. 3. Superposed curves are the magnetic-field lines, where the thick red curve depicts the outer border of the flux rope. The flux rope erupts at $t=4 \mathrm{hr}$. After another 7.5 hours, the velocity field and the magnetic field evolve to that shown in Fig. 3(b), where the flux-rope center is now at a height of $h=10 R_{\odot}$. From the figure, we can see that the ejecta of the CME is already at a height of approximately $8 R_{\odot}$. From Fig. 3(b), we can also see that, in the current sheet behind the CME, a blob develops (depicted by the white circle). In front of the flux rope, where the CME speed is the highest, we find a speed of about $370 \mathrm{~km} \mathrm{~s}^{-1}$, while the blob behind the flux rope moves more slowly at a speed of about $320 \mathrm{~km} \mathrm{~s}^{-1}$. As time evolves, Fig. 3(c) shows the evolution of the blobs for the next $4.5 \mathrm{~h}$. Ten frames are shown, from $t=11.5$ to $t=16 \mathrm{~h}$., with a 30 minute separation between them. In each frame, the width of the slide (i.e., the horizontal size) is $2.0 R_{\odot}$. At $t=12 \mathrm{~h}$., a second blob is formed. At $t=13.0 \mathrm{~h}$, the second blob moves up to $h=3.8 R_{\odot}$, and a third blob is formed at $h=2.8 R_{\odot}$. The third blob moves faster than the second, and, by $t=15 \mathrm{~h}$., they started to merge. The merging continues until $t=16 \mathrm{~h}$., when the center of the merged blob reaches a height of $h=8 R_{\odot}$ (with the top edge at $9 R_{\odot}$ and the lower end at $6.8 R_{\odot}$ ). The simulation results of the CME as well as the generation and evolution of the coalescing blobs are consistent with the STEREO observations of the 24 May 2010 event.

One signature of electron acceleration is a type-III radio burst. The left panel of Fig. 4 shows the radio observation from the WAVES instrument [37] that is onboard STEREO-A. Near 10:23, we see a type-III-like radio burst with a starting frequency of about $3.7 \mathrm{MHz}$ (corresponding to a density of $1.5 \times 10^{5} \mathrm{~cm}^{-3}$ ) and an ending frequency of about $2.01 \mathrm{MHz}$ (corresponding to a density of $0.5 \times 10^{5} \mathrm{~cm}^{-3}$ ). The radio burst lasts for approximately 6 minutes. The time resolution of the plot is $38 \mathrm{~s}$. Note that the starting time of the radio burst at 10:23 comes shortly after the reconnection between the blob and the overlying open magnetic field (which likely occurred between 10:09 

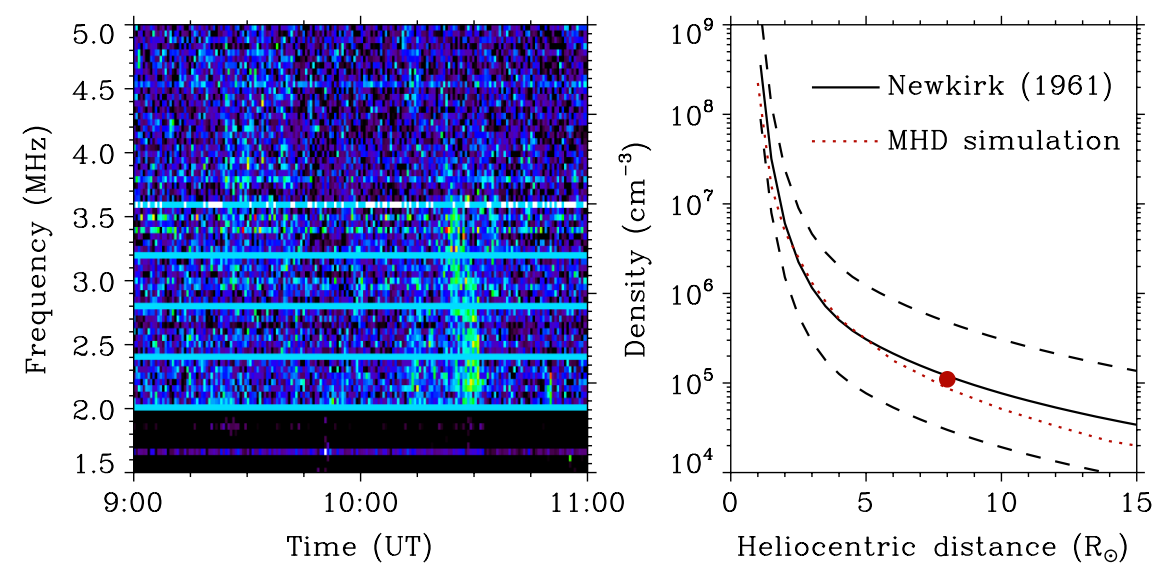

FIG. 4. Left: The observed type-III-like radio burst from STEREO-A. The starting frequency is approximately $3.7 \mathrm{MHz}$ at around 10:24. The ending frequency is not well discerned because there is no coverage of frequencies below 2 MHz. Right: Density of solar atmosphere as a function of height. The solid line is from the Newkirk model, with the two dashed lines enclosing it representing fourfold and one-quarter of the model value, respectively. The red dotted line is a realtime MHD calculation obtained using the Predictive Science STEREO modeling web site [46]. The red dot has a density of $10^{5} \mathrm{~cm}^{-3}$, corresponding to a frequency of approximately $3 \mathrm{MHz}$, and a height of $8 R_{\odot}$. These numbers agree with the location of the merged blob at 10:24.

and 10:24). This observation agrees with the fact that a type-III radio burst is caused by energetic electrons $(>10 \mathrm{keV})$ moving along open magnetic-field lines $[42,43]$. Note that the open-field-line geometry is important because, for such a geometry, a bump-in-tail distribution for electrons can quickly develop, which in turn can cause the excitation of Langmuir waves and, through nonlinear interaction, radio waves. For electrons moving in a closed blob, a bump-in-tail distribution can be hard to achieve, which may be the reason that no type-III radio bursts were observed prior to the opening-up process. We point out that, if the opening up started at around 10:09, then the type-III radio emission in our event did not occur right after the opening-up process started. This is because the generation of a type-III radio emission depends on the strength of the flux of the escaping electrons, which itself depends on the reconnection rate. The reconnection with the overlying open magnetic field is a slow process since it is controlled by the relative upward motion of the blob with respect to the plasma above it. Indeed, the reconnection electric field can be estimated as approximately $|\Delta U \times B| \simeq 0.15 \mathrm{~V} / \mathrm{m}$, where $\Delta U$ is the difference in speed between the blob and the plasma above it, and $B$ is the magnetic field at the top of the blob. We use $\Delta U \simeq$ $50 \mathrm{~km} / \mathrm{s}$ and approximately $0.03 \mathrm{G}$ from our numerical simulation.

Employing a density model, one can also infer the height of the blob when the radio burst occurs. The right panel of Fig. 4 plots the density profiles from two models. The solid black curve is the Newkirk density model [44]; the two dashed lines are the fourfold curve and the 1/4-fold curve, respectively. The red dotted line gives the radial profile of the plasma density at a latitude of $60^{\circ}$ and a longitude of $311^{\circ}$, from the global MHD simulation results [45] for the day of 23 May 2010 (prior to the CME eruption) provided by Predictive Science's STEREO modeling website [46]. The two models agree well and predict a height of $h \sim 8 R_{\odot}$ at $n=1.5 \times 10^{5} \mathrm{~cm}^{-3}$ [the red dot in the right panel of Fig. 4]. However, note that both the model of the Predictive Science group and the Newkirk model apply to undisturbed solar wind, which is almost certainly different from the solar wind of the post-CME current sheet.

At time 10:24, the top of the merged blob is at $h \sim 8 R_{\odot}$, consistent with the starting frequency of the observed type-III-like radio burst. Unfortunately, the WAVES instrument on STEREO-B was not working during this period. No radio signal was recorded by the Wind spacecraft at 1 A.U. This lack of signal at Wind could be due to the fact that the relative configuration of the CME and the Earth (head on) is different from the relative configuration of the CME and the two STEREO spacecraft (edge on). For a head-on configuration, where the CME propagates toward the observer, the densities along the line of sight are likely higher, and the radio signal may be absorbed or reflected.

We next discuss the electron-acceleration process. Since the splitting phase and the merging phase are fundamentally different, we consider them in turn. For the splitting phase, we argue that a first-order Fermi acceleration, similar to that proposed in Drake et al. [8], can exist even if the two daughter magnetic blobs expand slightly. For the merging phase, we follow the simulation of Oka et al. [15], in which the acceleration is due to electrons being trapped in the magnetic blobs; the electrons gain energies by experiencing the reconnecting electric field multiple times.

Consider first the splitting phase in which contraction of the blobs was identified. If the magnetic island has a size of $L$, then the electron acceleration rate can be estimated as [8] 


$$
d \epsilon / d t \sim \epsilon V_{A} / L
$$

where $\epsilon$ is the electron's energy, and $v_{A}$ is the Alfvén wave speed. Note, in the Drake et al. [8] scenario, if one assumes that the contraction is incompressible, then the area does not vary during contraction [47] and a net gain of particle energy requires the development of a large anisotropy of particle pitch angle [38]. The Drake et al. mechanism assumes that there is a reflection point at each end of the magnetic island. In our case, however, the splitting is going to lead to only one " $\mathrm{X}$ "-type reconnection point; there is no second reconnection point. Consequently, the electrons reflect only at the single $\mathrm{X}$ point, so $L$ in Eq. (1) should be replaced by $2 L$. In an expanding magnetic blob, the absence of the second reflection point is crucial for a net gain of energy. This is because, if the two reflection points are moving away from each other (due to the expansion), then the Fermi mechanism leads to a loss of energy (since the "collision" is head on, not tail on). If there is only one reflection point, then the Fermi mechanism still leads to an acceleration. At 07:09, the height of the mother blob is $r \sim 4.0 R_{\odot}$. Using an empirical solar wind model [48] with a magnetic field of $B=0.143 \mathrm{G}$ and a density of $2 \times 10^{5} \mathrm{~cm}^{-3}$, values appropriate at $4.0 R_{\odot}$, one finds an Alfvén wave speed of $V_{A}=700 \mathrm{~km} \mathrm{~s}^{-1}$. Behind the CME, we assume that $V_{A}$ does not differ much from its solar-wind value. Then, from Eq. (1) and a vertical scale of the daughter blob of approximately $2 L \simeq 0.6 R_{\odot}$, one obtains an acceleration time scale for a $10 \mathrm{keV}$ electron from an initial energy of $100 \mathrm{eV}$ to be approximately 5500 seconds or about $1.0 \mathrm{~h}$. This is more than 4 times longer than the duration of the splitting phase of $\leq 15$ minutes. Therefore, although electron acceleration likely occurred during the splitting phase, the acceleration may not be enough for the observed type-III radio burst.

Next, we consider the merging phase which started at 08:09. During this phase, there is no magnetic-island contraction. Instead, as the merging continued, the blob expands. However, Oka et al. [15] showed that nonthermal population of electrons can quickly develop from an initial thermal population when magnetic islands merge. We therefore consider below the acceleration time scale associated with the merging by using the simulation Oka et al. [15]. They found that the ratio of $n\left(10 T_{\mathrm{th}}\right) / n\left(T_{\mathrm{th}}\right) \sim 0.05$ and $n\left(100 T_{\text {th }}\right) / n\left(T_{\text {th }}\right) \sim 10^{-6}$ at $\Omega_{c i} t=115$, where $\Omega_{c i}=$ $e B / m_{i} c$ is the ion cyclotron frequency. The acceleration continues further, and one can infer from their Figure 4(a) that $n\left(100 T_{\text {th }}\right)$ doubles for a duration of $\Omega_{c i} t=13$. This calculation leads to $n\left(100 T_{\text {th }}\right) \sim 10^{-4} n\left(T_{\text {th }}\right)$ at $\Omega_{c i} t=200$. We note, however, that the simulation of Oka et al. [15] has a current-sheet width of $D \sim d_{i}$ and a length scale of the magnetic island of $l \sim 20 d_{i}\left(15<l / d_{i}<25\right.$ from their Figure 6), where $d_{i}=c / \omega$ is the ion inertial length. The blobs we consider here are of macroscopic scale of about
$1 R_{\odot}$ and are much larger than $d_{i}$ [49]. Nevertheless, we assume that the acceleration rate is inversely proportional to the time for an electron to travel around the blob. (See Figure 6 in Oka et al. [15] for typical trajectories of electrons, which are of the blob size, and the discussion in Drake et al. [8].) We further assume that the acceleration time scale can be estimated as $10^{-6}<n\left(100 T_{\text {th }}\right) / n\left(T_{\text {th }}\right)<$ $10^{-4}$. Then we can calculate the time for an electron to reach $10 \mathrm{keV}$ from an initial temperature of $T \sim 100 \mathrm{eV}$ (about $10^{6} \mathrm{~K}$ ), which is a 100 -fold increase in energy, to be $\left(115 / \Omega_{c i}\right) \times R_{\odot} / l<t<\left(200 / \Omega_{c i}\right) \times R_{\odot} / l$. With $R_{\odot}=$ $7 \times 10^{5} \mathrm{~km}, n=10^{5} \mathrm{~cm}^{-3}$ and a $B=0.1 \mathrm{G}$, we obtain $d_{i}=c /\left(1.32 \times 10^{3} n_{i}^{1 / 2}\right)=0.719 \mathrm{~km} \quad$ and $\quad \Omega_{c i}=(9580 \times$ $B / \mathrm{G})=958 \mathrm{~Hz}$. This yields the ratio of $\frac{\omega}{\Omega_{c i}}=4.33$, similar to the setup of $\frac{\omega}{\Omega_{c i}}=3.0$ used in [15]. Therefore, the acceleration time scale can be estimated to be $1.5 \mathrm{~h}$. $<$ $t<2.8 \mathrm{~h}$.

The assumption of a density of approximately $10^{5} \mathrm{~cm}^{-3}$ corresponds to a $3 \mathrm{MHz}$ type-III radio burst (as was observed). Our choice of a $B=0.1 \mathrm{G}$ is to yield an Alfvén wave speed of $700 \mathrm{~km} \mathrm{~s}^{-1}$. However, we note that the magnetic field at the reconnection site is subject to large uncertainties. In [19], Ko et al. examined the magnetic field in a current sheet behind a CME. At distances of $4 R_{\odot}$ and $5 R_{\odot}$, the inferred magnetic fields were 0.414 and $0.265 \mathrm{G}$, respectively, considerably higher than the quiet solar wind values. However, the blob studied in [19] was of higher speed (about $587 \mathrm{~km} \mathrm{~s}^{-1}$ ). In our 2.5-D axysymmetric MHD simulation where the blobs have comparable speeds, we obtained magnetic fields of 0.05 and $0.036 \mathrm{G}$ at 4 and $5 R_{\odot}$, which are approximately 5 times smaller than those reported in [19]. Obviously, the value of $B$ can affect the estimate of the acceleration time scale.

If there is some acceleration during the splitting phase, then the acceleration time scale of a $10 \mathrm{keV}$ electron associated with the merging $1.5 \mathrm{~h} .<t<2.8 \mathrm{~h}$. can be shorter. Our estimate of the acceleration time scale is comparable to the observation: The coalescence begins around 08:24, and the magnetic reconnection with the overlying magnetic-field lines occurs at around 10:24. Note that a "closed" geometry of the magnetic island can effectively confine energetic electrons. Therefore, even if electrons were accelerated above $10 \mathrm{keV}$ before 10:24, they could be held within the island until the magnetic island reconnected with the overlying field from above, after which electrons would be released to open field lines, and the classical generation mechanism for Langmuir waves can take place.

\section{SUMMARY}

In this paper, we report the first observational evidence of a macroscopic magnetic-field coalescence which occurred on 24 May 2010, along the current sheet behind a 
CME eruption. Data from STEREO-A, STEREO-B and SOHO spacecraft were analyzed. Before the coalescence, the first magnetic blob also underwent a splitting, although the duration of the splitting could not be determined given the limitation of a 15-minute time resolution of the data. The coalescence of the second magnetic blob with the first magnetic blob lasted long enough and can be seen from both the white light and the difference images. After the merging, the merged blob moved further upward until a reconnection occurred between the blob and the overlying magnetic-field lines that lie above the blob [best seen from the base-difference images shown in Fig. 2(f)]. Shortly after the inferred reconnection, a type-III-like radio burst was detected by the WAVES instrument on STEREO-A. (Unfortunately, the WAVES instrument on STEREO-B was not working during the observation window.) We suggest that the energetic electrons $(>10 \mathrm{keV})$ that are responsible for the detected type-III-like radio burst were mostly accelerated during magnetic-island coalescence through processes discussed in Oka et al. [15]. A preacceleration from an earlier splitting of the first magnetic island may have also occurred, through a first-order Fermi mechanism similar to that discussed in Drake et al. [8]. We have also examined the merging process using a 2.5-D axisymmetric MHD simulation of a catastrophic flux-rope model [39-41]. Multiple blobs were found in these simulations, and coalescence of blobs has been identified to occur along the current sheets behind the erupting flux rope (as well as above a nearby disturbed streamer). The modeled time scale of the blob propagation and coalescence, as well as the height of the coalescence, agree reasonably well with the observations.

Finally, we note that possibly three distinct magneticreconnection processes occurred in this event. Illustrated in the drawings of Fig. 2(g), these processes correspond to (1) splitting of the first blob into a "double-core-peanut" shape; (2) merging of the two blobs; and (3) reconnection between the merged magnetic island with the overlying open magnetic-field lines. The presence of the third reconnection process caused the accelerated electrons to be released to open magnetic field lines and led to the observed type-III-like radio burst-the signature of efficient electron acceleration; the acceleration presumably occurred in the first two reconnection processes.

Our study provides a useful basis for further theoretical investigations and numerical simulations of both the blob-generation mechanism and particle acceleration at coalescing magnetic islands.

\section{ACKNOWLEDGMENTS}

We thank the STEREO/WAVES instrument group for providing the 38-second radio data. This research is supported in part by 973 Program No. 2012CB825601 and NNSFC Grant Nos. 41104113, 40825014, and 40890162 for H. Q. S., Y. C., X. L. K., and S. W. F., and NNSFC Grant
No. 41028004 for Y. C. and G. L., at Shandong University, China; and NSF Early Career Grant No. ATM-0847719 and a NASA Grant NNX07AL52A (EpSCoR) for G. L. at the University of Alabama in Huntsville, USA. We also thank the two referees for their valuable suggestions.

[1] S. Masuda, T. Kosugi, H. Hara, S. Tsuneta, and Y. Ogawara, A Loop-Top Hard X-ray Source in a Compact Solar Flare as Evidence for Magnetic Reconnection, Nature (London) 371, 495 (1994).

[2] E. W. Hones, Jr., J. R. Asbridge, S. J. Bame, and S. Singer, Substorm Variations of the Magnetotail Plasma Sheet from $X_{\mathrm{SM}} \approx-6 R_{E}$ to $X_{\mathrm{SM}} \approx-60 R_{E}$, J. Geophys. Res. 78, 109 (1973).

[3] K. Hurley, S. E. Boggs, D. M. Smith, R. C. Duncan, R. Lin, A. Zoglauer, S. Krucker, G. Hurford, H. Hudson, C. Wigger et al., An Exceptionally Bright Flare from SGR 1806-20 and the Origins of Short-Duration $\gamma$-Ray Bursts, Nature (London) 434, 1098 (2005).

[4] C. J. Xiao, X. G. Wang, Z. Y. Pu, Z. W. Ma, H. Zhao, G. P. Zhou, J.X. Wang, M. G. Kivelson, S. Y. Fu, Z.X. Liu et al., Satellite Observations of Separator-Line Geometry of Three-Dimensional Magnetic Reconnection, Nature Phys. 3, 609 (2007).

[5] A. Retinò, D. Sundkvist, A. Vaivads, F. Mozer, M. André, and C.J. Owen, In Situ Evidence of Magnetic Reconnection in Turbulent Plasma, Nature Phys. 3, 235 (2007).

[6] R.P. Lin and H.S. Hudson, Non-thermal Processes in Large Solar Flares, Sol. Phys. 50, 153 (1976).

[7] R.P. Lin and RHESSI Team, Rhessi Observations of Particle Acceleration in Solar Flares, Adv. Space Res. 32, 1001 (2003).

[8] J. F. Drake, M. Swisdak, H. Che, and M. A. Shay, Electron Acceleration from Contracting Magnetic Islands During Reconnection, Nature (London) 443, 553 (2006).

[9] W. I. Axford, E. Leer, and G. Skadron in Proceedings of the Fifteenth International Cosmic Ray Conference (ICRC), Plovdiv, Bulgaria, 1977, Vol. 11 (Bulgarian Academy of Sciences, Sofia, 1977), p. 132.

[10] A. R. Bell, The Acceleration of Cosmic Rays in Shock Fronts. I, Mon. Not. R. Astron. Soc. 182, 147 (1978).

[11] A. R. Bell, The Acceleration of Cosmic Rays in Shock Fronts. II, Mon. Not. R. Astron. Soc. 182, 443 (1978).

[12] G. F. Krymskii, A Regular Mechanism for the Acceleration of Charged Particles on the Front of a Shock Wave, Dokl. Akad. Nauk. SSSR 234, 1306 (1977); [Sov. Phys. Dokl. 22, 327 (1977)].

[13] R. D. Blandford and J. P. Ostriker, Particle Acceleration by Astrophysical Shocks, Astrophys. J. 221, L29 (1978).

[14] X. R. Fu, Q. M. Lu, and S. Wang, The Process of Electron Acceleration During Collisionless Magnetic Reconnection, Phys. Plasmas 13, 012309 (2006).

[15] M. Oka, T.D. Phan, S. Krucker, M. Fujimoto, and I. Shinohara, Electron Acceleration by Multi-Island Coalescence, Astrophys. J. 714, 915 (2010).

[16] L. J. Chen, A. Bhattacharjee, P. A. Puhl-Quinn, H. Yang, N. Bessho, S. Imada, S. Mühlbachler, P. W. Daly, B. 
Lefebvre, Y. Khotyaintsev et al., Observation of Energetic Electrons within Magnetic Islands, Nature Phys. 4, 19 (2007).

[17] R.S. Wang, Q. M. Lu, A. M. Du, and S. Wang, In Situ Observations of a Secondary Magnetic Island in an Ion Diffusion Region and Associated Energetic Electrons, Phys. Rev. Lett. 104, 175003 (2010).

[18] M. Karlický, M. Bárta, and J. Rybák, Radio Spectra Generated During Coalescence Processes of Plasmoids in a Flare Current Sheet, Astron. Astrophys. 514, A28 (2010).

[19] Y. K. Ko, J. C. Raymond, J. Lin, G. Lawrence, J. Li, and A. Fludra, Dynamical and Physical Properties of a PostCoronal Mass Ejection Current Sheet, Astrophys. J. 594, 1068 (2003).

[20] J. Lin, Y. K. Ko, L. Sui, J. C. Raymond, G. A. Stenborg, Y. Jiang, S. Zhao, and S. Mancuso, Direct Observations of the Magnetic Reconnection Site of an Eruption on 2003 November 18, Astrophys. J. 622, 1251 (2005).

[21] P. Riley, R. Lionello, Z. Mikić, J. Linker, E. Clark, J. Lin, and Y.K. Ko, "Bursty" Reconnection Following Solar Eruptions: MHD Simulations and Comparison with Observations, Astrophys. J. 655, 591 (2007).

[22] Y. Chen, X. Li, H. Q. Song, Q. Q. Shi, S. W. Feng, and L.D. Xia, Intrinsic Instability of Coronal Streamers, Astrophys. J. 691, 1936 (2009).

[23] N.R. Sheeley, Jr., Y.M. Wang, S.H. Hawley, G.E. Brueckner, K.P. Dere, R. A. Howard, M. J. Koomen, C. M. Korendyke, D. J. Michels, S. E. Paswaters et al., Measurements of Flow Speeds in the Corona between 2 and 30 Rs., Astrophys. J. 484, 472 (1997).

[24] H. Q. Song, Y. Chen, K. Liu, S. W. Feng, and L. D. Xia, Quasi-periodic Releases of Streamer Blobs and Velocity Variability of the Slow Solar Wind near the Sun, Sol. Phys. 258, 129 (2009).

[25] H. Q. Song, X. L. Kong, Y. Chen, B. Li, G. Li, S. W. Feng, and L.D. Xia, A Statistical Study on the Morphology of Rays and Dynamics of Blobs in the Wake of Coronal Mass Ejections, Sol. Phys. 276, 261 (2011).

[26] N. R. Sheeley, Jr., H.P. Warren, and Y.M. Wang, The Origin of Postflare Loops, Astrophys. J. 616, 1224 (2004).

[27] D. E. McKenzie and S.L. Savage, Quantitative Examination of Supre-Arcade Downflows in Eruptive Solar Flares, Astrophys. J. 697, 1569 (2009).

[28] S. L. Savage, D. E. McKenzie, and K. K. Reeves, ReInterpretation of Supra-Arcade Downflows in Solar Flares, Astrophys. J. Lett. 747, L40 (2012).

[29] R. A. Howard, J. D. Moses, A. Vourlidas, J. S. Newmark, D. G. Socker, S. P. Plunkett, C. M. Korendyke, J. W. Cook, A. Hurley, J.M. Davila et al., Sun Earth Connection Coronal and Heliospheric Investigation (SECCHI), Space Sci. Rev. 136, 67 (2008).

[30] M. L. Kaiser, T. A. Kucera, J. M. Davila, O. C. St. Cyr, M. Guhathakurta, and E. Christian, The STEREO Mission: An Introduction, Space Sci. Rev. 136, 5 (2007).

[31] A. Ciaravella, J.C. Raymond, J. Li, P. Reiser, L. D. Gardner, Y.K. Ko, and S. Fineschi, Elemental Abundances and Post-coronal Mass Ejection Current
Sheet in a Very Hot Active Region, Astrophys. J. 575, 1116 (2002).

[32] See the material at http://staff.ustc.edu.cn/ yaochen/ blobs.html.

[33] Computer-Aided CME Tracking or CACTUS, http://sidc .oma.be/cactus.

[34] E. Robbrecht and D. Berghmans, Automated Recognition of Coronal Mass Ejections (CMEs) in Near-Real-Time Data, Astron. Astrophys. 425, 1097 (2004).

[35] E. Robbrecht, D. Berghmans, and R. A. M. Van der Linden, Automated LASCO CME Catalog for Solar Cycle 23: Are CMEs Scale Invariant?, Astrophys. J. 691, 1222 (2009).

[36] C. J. Schrijver and M.L. DeRosa, Photospheric and Heliospheric Magnetic Fields, Sol. Phys. 212, 165 (2003).

[37] J. L. Bougeret, K. Goetz, M. L. Kaiser, S. D. Bale, P. J. Kellogg, M. Maksimovic, N. Monge, S. J. Monson, P. L. Astier, S. Davy et al., S/WAVES: The Radio and Plasma Wave Investigation on the STEREO Mission, Space Sci. Rev. 136, 487 (2008).

[38] J. F. Drake, M. Opher, M. Swisdak, and J. N. Chamoun, A Magnetic Reconnection Mechanism for the Generation of Anomalous Cosmic Rays, Astrophys. J. 709, 963 (2010).

[39] Y.Q. Hu, G.Q. Li, and X. Y. Xing, Equilibrium and Catastrophe of Coronal Flux Ropes in Axisymmetrical Magnetic Field, J. Geophys. Res. 108, 1072 (2003).

[40] Y. Chen, G. Q. Li, and Y. Q. Hu, Force Balance Analysis of a Coronal Magnetic Flux Rope in Equilibrium of Eruption, Astrophys. J. 649, 1093 (2006).

[41] Y. Chen, Y. Q. Hu, and S. J. Sun, Catastrophic Eruption of Magnetic Flux Rope in the Corona and Solar Wind with and without Magnetic Reconnection, Astrophys. J. 665, 1421 (2007).

[42] G. Mann and A. Klassen, Electron Beams Generated by Shock Waves in the Solar Corona, Astron. Astrophys. 441, 319 (2005).

[43] R. E. Ergun, D. Larson, R. P. Lin, J. P. McFadden, C. W. Carlson, K. A. Anderson, L. Muschietti, M. McCarthy, G. K. Parks, H. Reme et al., Wind Spacecraft Observations of Solar Impulsive Electron Events Associated with Solar Type III Radio Bursts, Astrophys. J. 503, 435 (1998).

[44] G. J. Newkirk, The Solar Corona in Active Regions and the Thermal Origin of the Slowly Varying Component of Solar Radio Radiation, Astrophys. J. 133, 983 (1961).

[45] P. Riley, J. A. Linker, and Z. Mikić, An Empirically-Driven Global MHD Model of the Solar Corona and Inner Heliosphere, J. Geophys. Res. 106, 15889 (2001).

[46] See the data at http://www.predsci.com/stereo.

[47] R. L. Fermo, J. F. Drake, and M. Swisdak, A Statistical Model of Magnetic Islands in a Current Layer, Phys. Plasmas 17, 010702 (2010).

[48] M. Guhathakurta, E. C. Sittler, and D. McComas, Semiempirical MHD Model of the Solar Wind and Its Comparison with Ulysses, Space Sci. Rev. 87, 199 (1999).

[49] $\omega=\left(4 \pi n_{i} Z^{2} e^{2} / m_{i}\right)^{1 / 2}$ is the ion plasma frequency, with $Z$ and $m_{i}$ the ion charge and mass, respectively. 